\title{
Juvenile dermatomyositis forty years on: Case report
}

\author{
Inês Rego de Figueiredo ${ }^{\mathrm{a}, 1, *}$, Sara Guerreiro Castro ${ }^{\mathrm{a}}$, Vera Bernardino ${ }^{\mathrm{a}}$, José Silva Nunes ${ }^{\mathrm{b}}$, \\ Pedro Alves ${ }^{\mathrm{c}}$, Maria Francisca Moraes-Fontes ${ }^{\mathrm{a}}$ \\ ${ }^{a}$ Unidade de Doenças Auto-imunes/Medicina 7.2, Hospital de Curry Cabral, Centro Hospitalar de Lisboa Central (CHLC), Portugal \\ ${ }^{\mathrm{b}}$ Serviço de Endocrinologia, Hospital de Curry Cabral, CHLC, Portugal

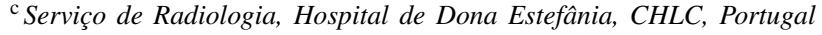

Received 13 November 2017; received in revised form 10 April 2018; accepted 26 June 2018

\begin{abstract}
We present a case report of a 42 year old female, diagnosed at the age of 3 with Juvenile Dermatomyositis. The clinical course was severe and refractory to immunosuppressive therapy. Currently, she is mostly affected by severe muscle atrophy, large joint contractures, calcinosis, and a lipodystrophy associated metabolic syndrome with hypertriglyceridemia, insulin resistance, high total testosterone and hepatic steatosis. She developed Hodgkinś lymphoma in the course of her disease. Personalized therapeutic choices are discussed as regards juvenile dermatomyositis complications.
\end{abstract}

(C) 2018 Elsevier B.V. All rights reserved.

Keywords: Juvenile dermatomyositis; Lipodystrophy; Calcinosis.

\section{Introduction}

Juvenile dermatomyositis (JDM) is an autoimmune disease resulting in perivascular inflammation, perifascicular atrophy and muscle degeneration [1,2] and represents $85 \%$ of the idiopathic inflammatory myopathies in childhood [3]. Novel autoantibodies associated to specific clinical phenotypes have been described in the past decade. Amongst these, anti-p155/140 - targeted to transcriptional intermediary factor 1 gamma (TIF1- $\gamma$ ) and anti-p140 - targeted to nuclear matrix protein 2 (NXP2), are more likely to occur in children with calcinosis and cutaneous ulceration [4-6].

Fifty years ago, active treatment of childhood onset JDM resulted in much improved prognosis [7]. Despite progress, standard of care still remains confined to untargeted immunosuppressive therapy with steroids [8]. As recently reviewed [9], the use of steroid sparing agents is recommended, usually with methotrexate, but also azathioprine and cyclosporine,

\footnotetext{
* Corresponding author.

E-mail address: inesregofigueiredo@gmail.com (I. Rego de Figueiredo).

1 Permanent address: Unidade de Doenças Auto-imunes/Medicina 7.2, Hospital de Curry Cabral, Rua da Beneficência, nº 8, 1069-166 Lisboa, Portugal.
}

intravenous immune globulin, tacrolimus, rituximab and cyclophosphamide in refractory cases. Cardiac or respiratory involvement lower 10-year survival rates, otherwise reported to be over $90 \%[10,11]$. Many children therefore survive to adulthood, but there are scarce descriptions of disease activity, co-morbidities and functional status after prolonged disease. Aiming to contribute to disease knowledge we report an adult patient with JDM exhibiting lipodystrophy, ongoing calcinosis, and irreversible joint contractures, in whom a prior diagnosis of malignancy restricts therapeutic choices and whose management remains an ongoing challenge.

\section{Case report}

The female patient, currently 42 years-old, was admitted to the Hammersmith Hospital in London, at the age of 3. At that time, major complaints were proximal muscle weakness with Gowers sign, unilateral facial nerve palsy and ulcerating skin lesions (Fig. 1a-d). The electromyography of right deltoid, triceps and tibialis anterior muscles revealed short, small amplitude polyphasic potentials, with no spontaneous activity at rest. The diagnosis of JDM was made on the basis of needle biopsy of left quadriceps displaying vacuolar 


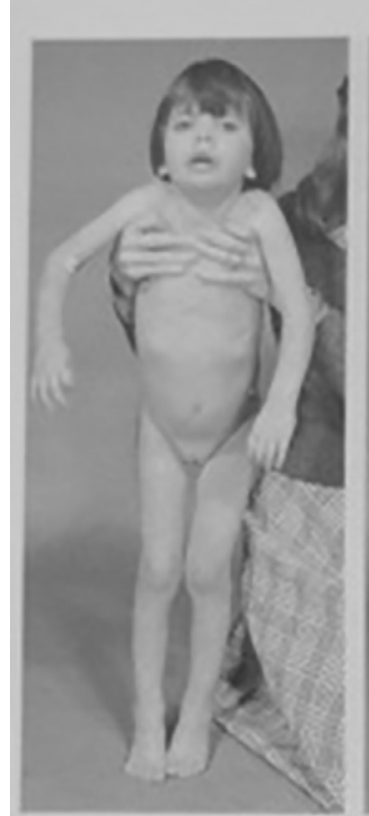

(a)

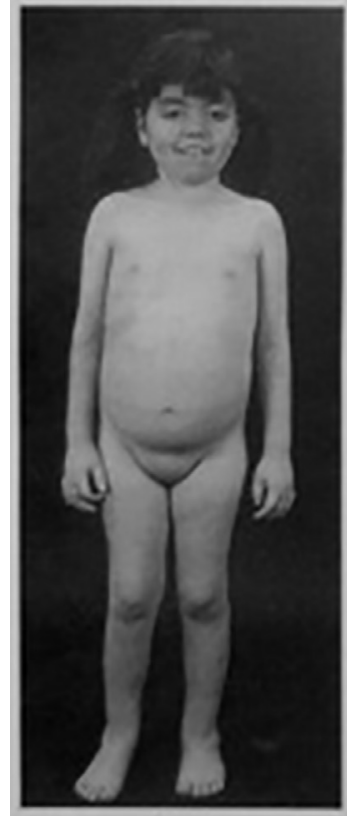

(e)

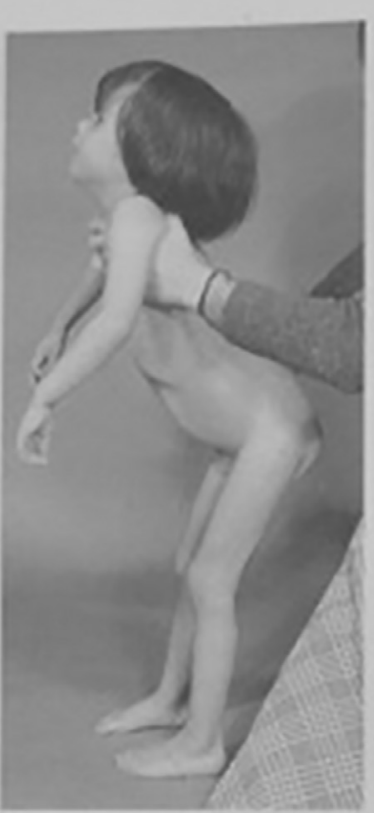

(b)

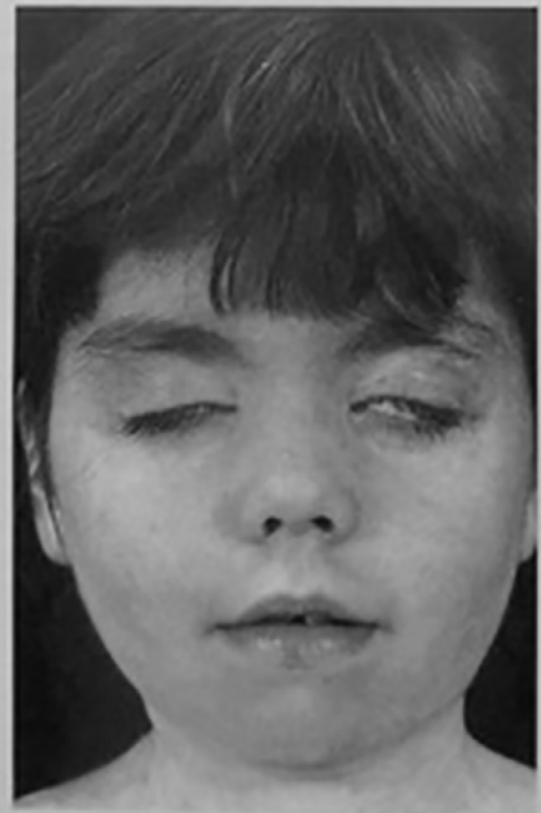

(f)

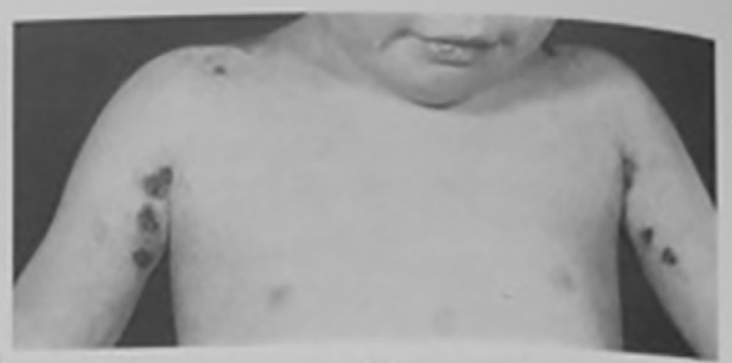

(c)

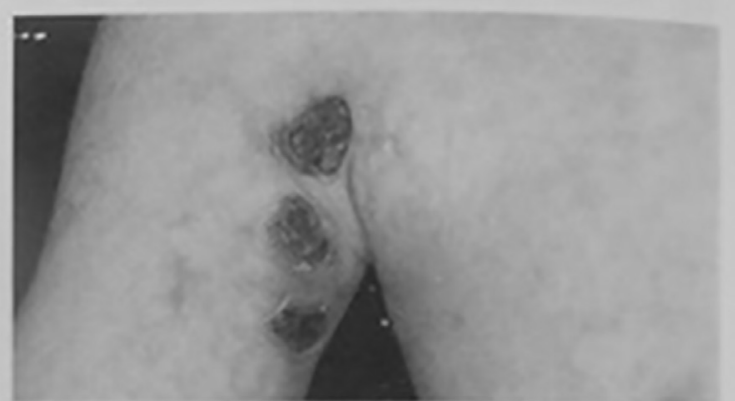

(d)

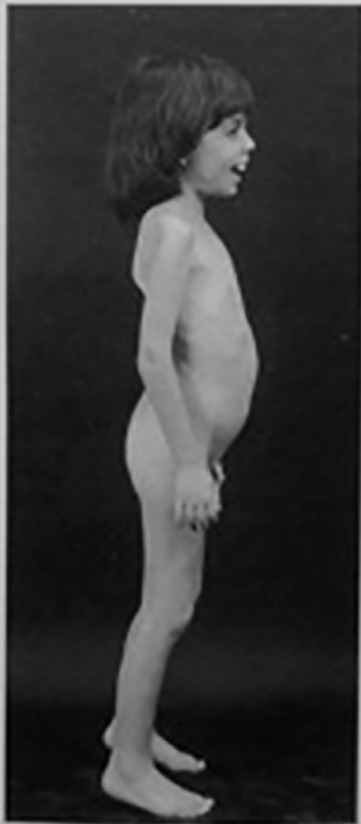

(g)

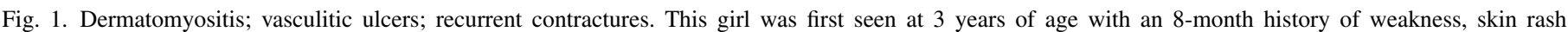

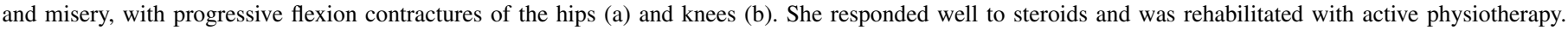

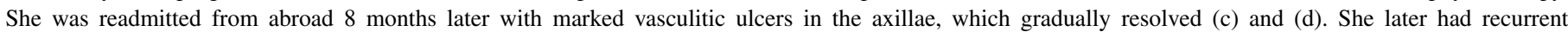

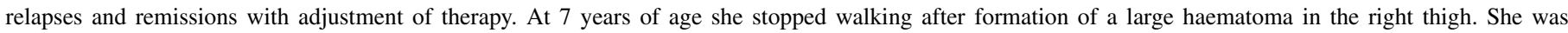

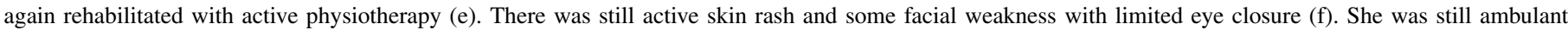

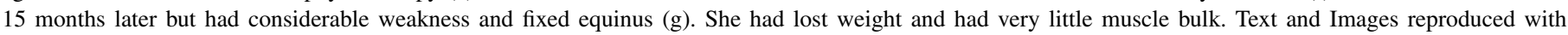
permission from Muscle Disorders in Childhood, 2nd Ed. By V. Dubowitz. 549 pp., illustrated. Philadelphia: W.B. Saunders, 1995.

appearance of several muscle fibers and perifascicular atrophy (Supplementary Fig. 1).

From the time of diagnosis, treatment consisted of a steroid regimen, initially given alone and subsequently combined with azathioprine, followed by cyclosporine. From the onset of the illness, periods of immobility were followed by major joint contractures and over the next few years she was regularly admitted to hospital for periods of intensive physiotherapy which were extremely successful (Fig. 1e-g). Of note, there was no history of consanguinity, episodic fevers, seizures, anemia, respiratory difficulties or learning disabilities and she successfully completed a university degree. 
Overall, response to treatment was poor and by the age of 21 she was wheelchair bound and immunosuppressive therapy was stopped. Even though the episodes of cutaneous ulceration no longer occurred, several other features developed over the next years namely an erythematous pruritic and progressively more indurated facial skin, generalized calcinosis and intermittent diarrhea, the latter attributed to intestinal bacterial overgrowth syndrome. Nodular-Sclerosis Classical Hodgkin Lymphoma (stage II, supra-diaphragmatic, category B) was diagnosed at age 31, with full remission after six cycles of chemotherapy consisting of doxorubicin, bleomycin, vinblastin and dacarbazine. There was no serological evidence of acute Epstein-Barr virus infection (virological status in lymphoma cells was not evaluated). She did not tolerate a cyproterone/ethinylestradiol combination pill due to dysmenorrhoea.

At the age of 38 she was re-evaluated in our Autoimmune Diseases Unit. She complained of menstrual irregularities, hirsutism and relentless calcinosis. She exhibited low stature, diffuse alopecia, poikiloderma in a photosensitive distribution, symmetric parotid hypertrophy, periungueal telangiectasia, livedo reticularis (over the trunk and anterior thighs) and multiple atrophic scars corresponding to areas of previous ulceration. There were fixed contractures of the major joints (shoulders, elbows and knees), lumbar scoliosis, generalized muscle atrophy and multiple foci of calcinosis along the muscle fascia of limbs and trunk. The abdomen was disproportionately large and corresponded to a most striking lipodystrophy with absence of fat in the face and limbs (Fig. 2). There was no cervical weakness, no difficulty in speaking or in swallowing. She was able to move her upper limbs, pick up small objects and feed herself.

Laboratory evaluation revealed normal creatine kinase and aldolase, increased total serum testosterone of $71 \mathrm{ng} / \mathrm{dL}$, (normal values $10,8-56,9 \mathrm{ng} / \mathrm{dL}$ ), but normal levels of serum thyroid stimulating hormone, prolactin, androstenedione, basal 17-hydroxy progesterone, estradiol, FSH, LH, ACTH and serum cortisol levels. Her fasting plasma glucose $(69 \mathrm{mg} / \mathrm{dL})$ and Peptide C $(6,5 \mathrm{ng} / \mathrm{mL}$, normal range $0,9-7.1 \mathrm{ng} / \mathrm{mL})$ were normal but insulin was increased to $65,5 \mathrm{uUI} / \mathrm{mL}$ (normal range 1,9-23 uUI/mL). She also had hypertriglyceridemia $(1860 \mathrm{mg} / \mathrm{dL}$, normal range $<150 \mathrm{mg} / \mathrm{dL})$ and low levels of high density lipoprotein cholesterol $(28 \mathrm{mg} / \mathrm{dL}$, normal range $<130 \mathrm{mg} / \mathrm{dL}$ ). Liver transaminases were normal but the gamma-glutamyl transferase was 3 fold elevated; ANA testing was intermittently positive, with a low title (1/160) but no specific positivity was found; there was no lung parenchymal change in the chest radiograph which displayed multiple calcifications in the subcutaneous tissues; the echocardiogram was normal; no structural abnormality or pressure changes were detected by Doppler echocardiography and nailfold video-capilaroscopy revealed a late-scleroderma pattern (Supplementary Fig. 2). Magnetic resonance imaging (MRI) showed diffuse muscle fatty atrophy (Grade III-IV) of masseters, shoulder girdle, deltoids, paravertebral thoracic, gluteal and thigh groups. The only relatively preserved muscles localized to the neck, thighs (adductor magnus and vastus in- termedius) and legs (tibialis anterior and posterior and toe extensors). There was no sign of muscle inflammation on fluid sensitive sequences (STIR and T2-weighted images). Parotid glands were heterogeneous with fatty infiltrates and diffuse calcifications. There was a marked increase in posterior cervical, axillary, mediastinal and intra-peritoneal fat deposition, also asymmetrically localized to the anterior and lateral portion of both thighs (Fig. 3). Small areas of subcutaneous oedema were suggestive of panniculitis in thighs and legs. Hepatomegaly was noted with splenomegaly, normal kidneys, uterus and ovaries. There was no lymphadenopathy. No mutations were found in the genes LMNA, ZMPSTE24, PTRF, CAV-1, AGPAT2, BSCL2, PPARG, INSR, PLIN1, CIDEC, PIK3R1, NSMCE2, POC1A, PCYT1A, POLD1 or PSMB8. Soon after the genetic tests were performed, the patientś serum tested positive for anti-transcriptional intermediary factor gamma protein antibody (TIF1-gamma) (Euroimmun ${ }^{\circledR}$ Lübeck, Euroline scan software, patient intensity 15, control 99), supporting the original diagnosis. There was no reactivity against the following antigens: Mi-2 (nucleosome remodeling deacetyalse complex), MDA (melanoma-differentiation associated gene 5), SRP (54kDa, signal recognition particle), NXP-2, SAE (small-ubiquitin-like modifier activating enzyme), Ku, PM-Scl (75 and $100 \mathrm{kDa}$ ), Jo-1 (histidyl-tRNA synthetase), PL-7 (threonyl-tRNA synthetase), PL-12 (alanyltRNA synthetase), EJ protein (glycyl-tRNA synthetase), OJ (isoleucyl-tRNA synthetase) and Ro-52KD.

Following re-assessment, weekly s.c. methotrexate (up to $20 \mathrm{mg} /$ week) and quarterly i.v. Pamidronate failed to have a positive impact on the rate of calcinosis deposits and range of metabolic abnormalities. The latter was associated with an episode of renal colic and both were discontinued after two years. Since then, painful calcium deposits have been removed surgically on three occasions. She has not tolerated metformin due to side-effects and is poorly compliant with fenofibrate. Decreased gastrointestinal absorption may also contribute to a poor response to therapy and serum triglyceride concentrations are usually 5-6 fold elevated. She reports beneficial anti-diarrheal effects with VSL\# $3^{\circledR}$, a commercially available probiotic, taken as required. Apart from feeling that the skin around her face is tighter there has been no change in her clinical features over the past four years. She is partially dependent for her hygiene needs which restrict her social life but is otherwise independent in her daily activities with an electric wheelchair and adapted motorized vehicle. She undertakes physiotherapy sessions consisting of passive limb mobilization three times per week.

\section{Discussion}

JDM is a very rare condition with an incidence of 2-4 cases per million per year, mostly affecting females. In so much as the peak incidence occurs between the ages of 510 , up to $25 \%$ of patients experience disease onset before the age of 4 [12], similarly to our patient. The disease may remit, relapse or follow a chronic continuous form [13]. When 


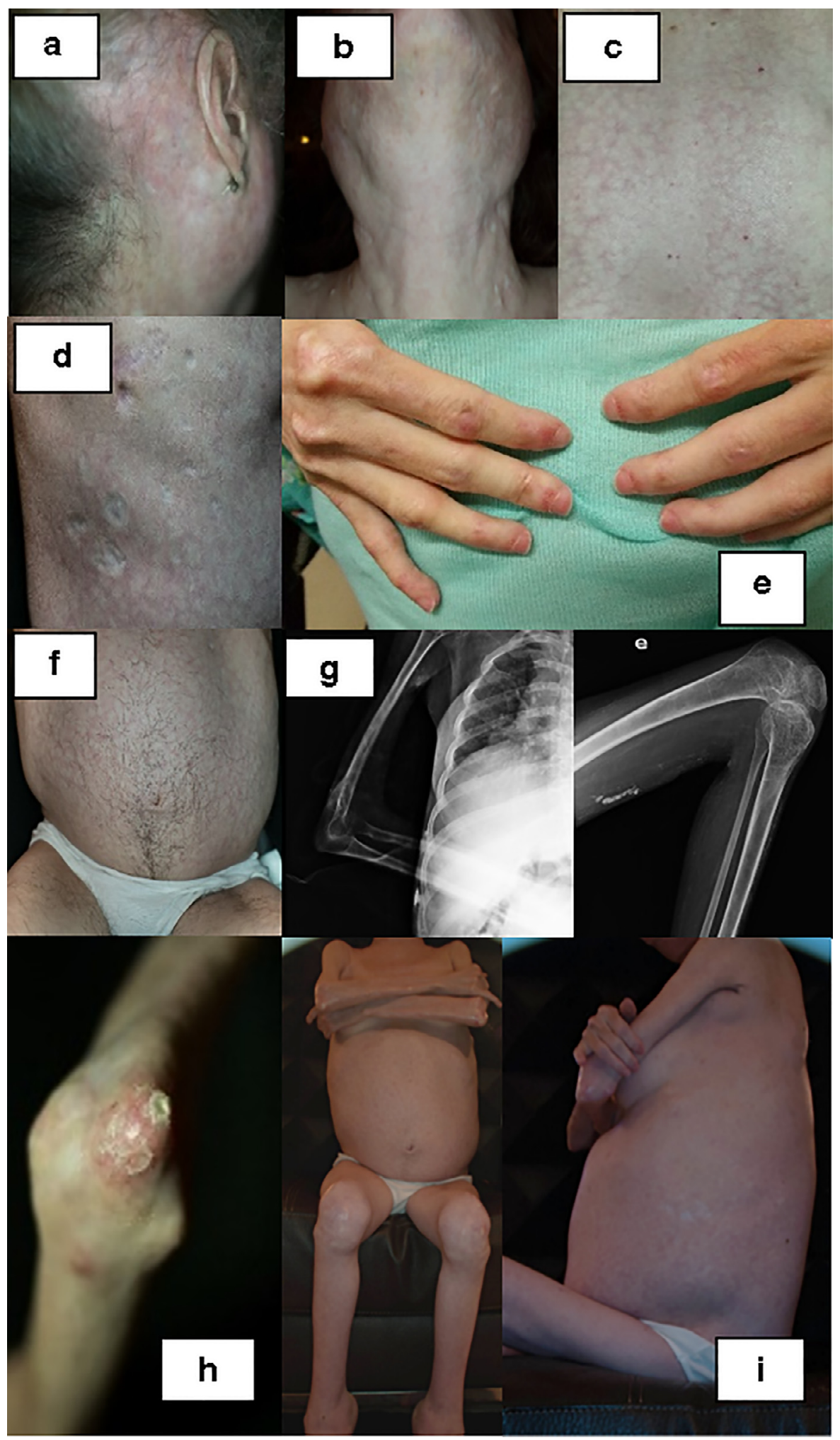

Fig. 2. Clinical features of the patient as an adult: poikiloderma and skin tightness (a); parotid hypertrophy (b); livedo reticularis over dorsum (c); atrophic scars (d); periungueal telangiectasia (e); hirsutism (f); calcinosis in fascial planes and joint contractures (g); cutaneous calcinosis (h); lipodystrophy with abdominal redistribution of fat and muscle atrophy (i). 

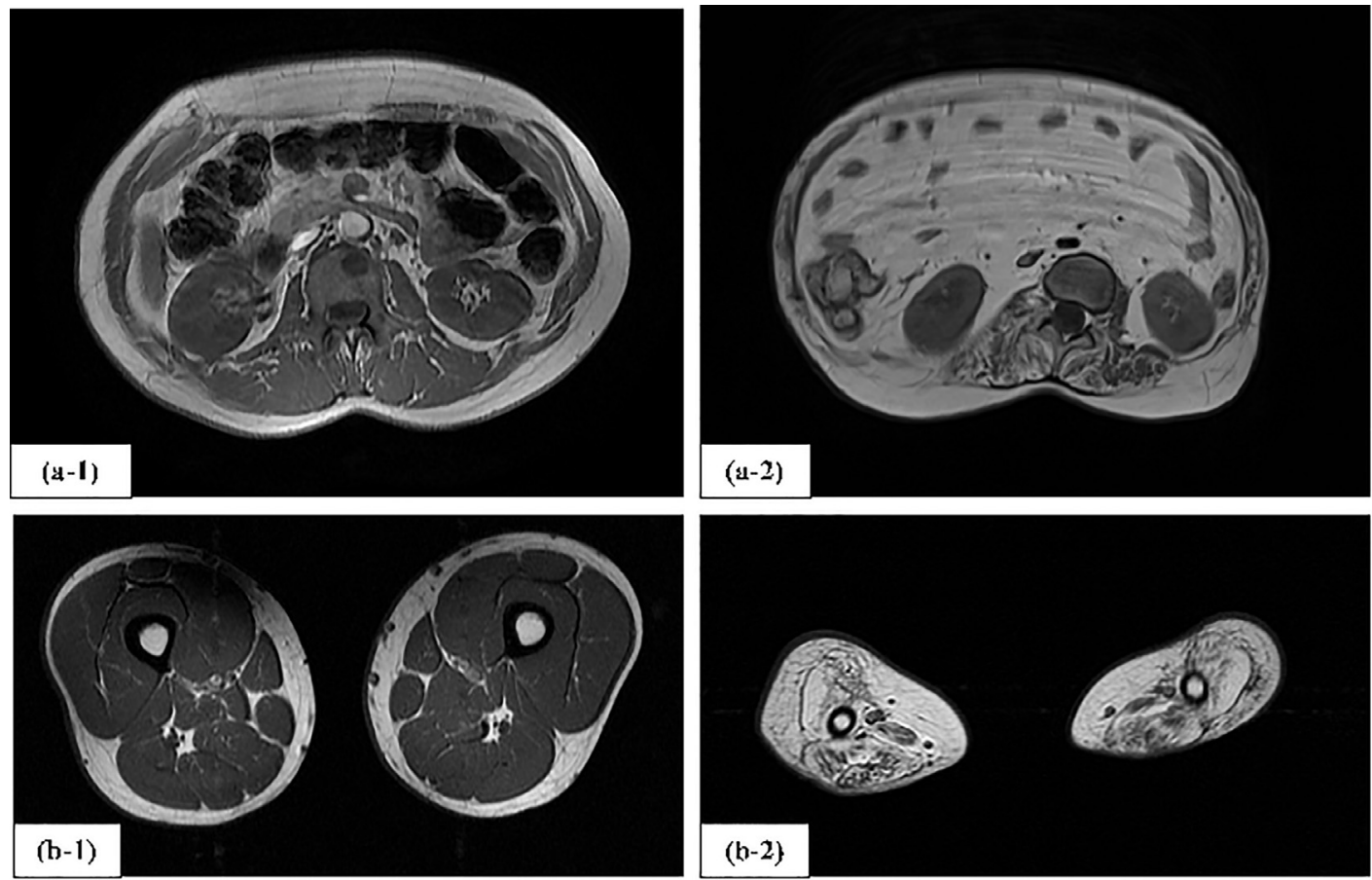

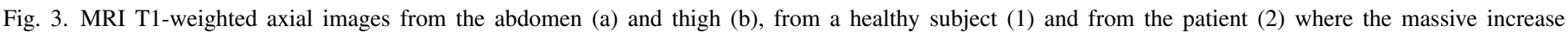

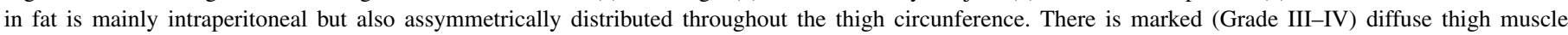
atrophy.

compared with adult auto-antibody reactivities, anti-TIF1- $\gamma$ positive children are more frequently affected by muscle atrophy, contractures, calcinosis and cutaneous vasculitis [14]. Of note, our patient received appropriate therapy, namely steroids and cyclosporine from disease onset. Physiotherapy treatments were intense but intermittent, with prolonged periods of immobility. Recurrent contractures developed from early on in the course of disease.

Several complex complications developed as disease progressed. Lymphoma developed in adulthood, many years after disease onset. In a recent literature review lymphoma was very rarely diagnosed in juvenile dermato/polymyositis, occurred only after the disease was well established and all the patients suffered from the non-Hodgkinś lymphoma type [15]. Hodgkin lymphoma (HL) has been described in non-immunosuppressed individuals but HL-like conditions have also been described in the spectrum of post-transplant lymphoproliferative disorders [16]. After a ten year-lapse, a relationship to prior immunosuppressive therapy remains an elusive possibility in our patient.

Albeit not confirmed, the initial lack of an auto-antibody marker, the presence of marked lipodystrophy, possible panniculitis and lack of muscle inflammation in the biopsy specimen reviewed raised the suspicion of an alternative diagnosis but no evidence of an auto-inflammatory disorder or a muscle dystrophy was found. While the lipodystrophy was suggestive of Chronic Atypical Neutrophilic Dermatosis with Lipodystrophy and Elevated temperatures (CANDLE) [17,18], lack of periodic fevers and systemic inflammation made this less likely, whereas the presence of anti-TIF1- $\gamma$, muscle atrophy, joint contractures, calcinosis and nailfold changes clinched the diagnosis of JDM.

JDM occurs in genetically susceptible patients [19-22]. Its association with lipodystrophy (deposition of fat in ectopic locations such as in salivary glands, the peritoneal cavity, liver and muscle and absence of fat deposits in other sites) [23,24] and lipodystrophy-associated metabolic abnormalities (hypertriglyceridemia, insulin resistance, high testosterone, hypertrichosis and probable fatty liver disease) has been described with severe calcinosis, joint contractures, muscle atrophy, chronic continuous illness course, facial erythema and anti-TIF1- $\gamma$ positivity $[25,26]$, all of which were present in our patient.

Follow up of JDM patients into adulthood has demonstrated the overall systemic nature of the disease and its impact on several organs on the long term. Even though studies on a Norway cohort followed over 16,8 years (2-38,1 years) has showed JDM patients often maintain active disease in the long term, our patient mostly suffers from cumulative organ damage, whose main predictor is disease activity at onset [27].

Because of the loss of adipose tissue, levels of the adipocyte-secreted hormone leptin may be low and leptin replacement could be a therapeutic option in our patient, in an attempt to overcome morphological and metabolic abnormalities. However lymphoma after leptin therapy for patients with acquired lipodystrophy has been described [28]. In addition, no single drug or associations of drugs has been effective in 
the treatment of calcinosis in JDM [29]. Upregulation of type I interferon pathway, described as a biomarker of JDM disease activity [30] as well as an effector molecule in CANDLE [31], may be a driver of disease pathogenesis. It is tempting to speculate that antagonizing type I interferon could have a favorable therapeutic impact in our patient. Overall, a history of lymphoma, longstanding immune-mediated disease, a potentially vulnerable immune system and the degree of irreversible organ damage lead us to take a conservative "do no harm" approach.

In conclusion we present a patient with a diagnosis of JDM at the age of 3 years, with a partial response to therapy and several co-morbidities. Marked lipodystrophy and little inflammation in the original muscle biopsy specimen raised suspicion of an overlap with muscle dystrophy which was not confirmed. Our key message highlights the extraordinary advances introduced in the 1980s, reducing patient mortality and side-effects of extremely high dosages of steroids. Despite the presence of disability our patient survived a severe childhood disease and leads a meaningful life.

\section{Acknowledgments}

Victor Dubowitz for diagnosis and clinical care from diagnosis until adulthood, clinical information, images and discussions, Vanda Lúcio Bernardes for physiotherapy, Francesco Muntoni, Ana Grilo, Ana Catarina Rodrigues, Manuel Vaz Riscado, Nuno Risoand António Panarra for clinical care, António Caetano for imaging, Caroline Sewry for histopathology review, Jocelyne Demengeot, Jocelyne Magré, Corinne Vigouroux, Olivier Lascols, Pascale Richard, Annachiara De Sandre-Giovannoli and Nuno Costa for genetic testing, Maria Céu Santos for auto-antibody profiling and W. B. Saunders (now Elsevier) publishers for permission to use images of the patient as a child [1].

\section{Supplementary materials}

Supplementary material associated with this article can be found, in the online version, at doi:10.1016/j.nmd.2018.06. 011 .

\section{References}

[1] Dubowitz V. Muscle disorders in childhood. 2nd ed. W.B. Saunders (now Elsevier); 1995.

[2] Feldman BM, Rider LG, Reed AM, Pachman LM. Juvenile dermatomyositis and other idiopathic inflammatory myopathies of childhood. Lancet 2008;371(9631):2201-12.

[3] McCann LJ, Juggins AD, Maillard SM, Wedderburn LR, Davidson JE, Murray KJ, et al. The Juvenile Dermatomyositis National Registry and Repository (UK and Ireland)-clinical characteristics of children recruited within the first 5yr. Rheumatology 2006;45(10):1255-60.

[4] Betteridge ZE, Gunawardena H, McHugh NJ. Novel autoantibodies and clinical phenotypes in adult and juvenile myositis. Arthritis Res Ther. 2011;13(2):209.

[5] Gunawardena H, Betteridge ZE, McHugh NJ. Myositis-specific autoantibodies: their clinical and pathogenic significance in disease expression. Rheumatology 2009;48(6):607-12.
[6] Gunawardena H, Wedderburn LR, Chinoy H, Betteridge ZE, North J, Ollier WE, et al. Autoantibodies to a $140-\mathrm{kd}$ protein in juvenile dermatomyositis are associated with calcinosis. Arthritis Rheum 2009;60(6):1807-14.

[7] Miller G, Heckmatt JZ, Dubowitz V. Drug treatment of juvenile dermatomyositis. Arch Dis Child 1983;58(6):445-50.

[8] Dubowitz V. Treatment of dermatomyositis in childhood. Arch Dis Child 1976;51(7):494-500.

[9] Malik A, Hayat G, Kalia JS, Guzman MA. Idiopathic Inflammatory Myopathies: Clinical Approach and Management. Front Neurol. 2016;7:64.

[10] Danko K, Ponyi A, Constantin T, Borgulya G, Szegedi G. Long-term survival of patients with idiopathic inflammatory myopathies according to clinical features: a longitudinal study of 162 cases. Medicine 2004;83(1):35-42.

[11] Peloro TM, Miller OF, Hahn TF 3rd, Newman ED. Juvenile dermatomyositis: a retrospective review of a 30-year experience. J Am Acad Dermatol 2001;45(1):28-34.

[12] Symmons DP, Sills JA, Davis SM. The incidence of juvenile dermatomyositis: results from a nation-wide study. $\mathrm{Br} \mathrm{J}$ Rheumatol 1995;34(8):732-6.

[13] Constantin T, Ponyi A, Orbán I, Molnár K, Dérfalvi B, Dicso F, et al. National registry of patients with juvenile idiopathic inflammatory myopathies in Hungary - clinical characteristics and disease course of 44 patients with juvenile dermatomyositis. Autoimmunity 2006;39(3):223-32.

[14] Espada G, Maldonado Cocco JA, Fertig N, Oddis CV. Clinical and serologic characterization of an Argentine pediatric myositis cohort: identification of a novel autoantibody (anti-MJ) to a $142-\mathrm{kDa}$ protein. J Rheumatol 2009;36(11):2547-51.

[15] Stubgen JP. Juvenile dermatomyositis/polymyositis and lymphoma. J Neurol Sci 2017;377:19-24.

[16] Carbone A, Spina M, Gloghini A, Tirelli U. Classical Hodgkin's lymphoma arising in different host's conditions: pathobiology parameters, therapeutic options, and outcome. Am J Hematol 2011;86(2):170-9.

[17] Torrelo A, Patel S, Colmenero I, Gurbindo D, Lendinez F, Hernan$\operatorname{dez} \mathrm{A}$, et al. Chronic atypical neutrophilic dermatosis with lipodystrophy and elevated temperature (CANDLE) syndrome. J Am Acad Dermatol 2010;62(3):489-95.

[18] Liu Y, Ramot Y, Torrelo A, Paller AS, Si N, Babay S, et al. Mutations in proteasome subunit beta type 8 cause chronic atypical neutrophilic dermatosis with lipodystrophy and elevated temperature with evidence of genetic and phenotypic heterogeneity. Arthritis Rheum 2012;64(3):895-907

[19] Reed AM, Pachman L, Ober C. Molecular genetic studies of major histocompatibility complex genes in children with juvenile dermatomyositis: increased risk associated with HLA-DQA1 *0501. Hum Immunol 1991;32(4):235-40.

[20] Mamyrova G, O’Hanlon TP, Monroe JB, Carrick DM, Malley JD, Adams S, et al. Immunogenetic risk and protective factors for juvenile dermatomyositis in Caucasians. Arthritis Rheum 2006;54(12):3979-87.

[21] Pachman LM, Liotta-Davis MR, Hong DK, Kinsella TR, Mendez EP, Kinder JM, et al. TNFalpha-308A allele in juvenile dermatomyositis: association with increased production of tumor necrosis factor alpha, disease duration, and pathologic calcifications. Arthritis Rheum 2000;43(10):2368-77.

[22] Rider LG, Artlett CM, Foster CB, Ahmed A, Neeman T, Chanock SJ, et al. Polymorphisms in the IL-1 receptor antagonist gene VNTR are possible risk factors for juvenile idiopathic inflammatory myopathies. Clin Exp Immunol 2000;121(1):47-52.

[23] Quecedo E, Febrer I, Serrano G, Martinez-Aparicio A, Aliaga A. Partial lipodystrophy associated with juvenile dermatomyositis: report of two cases. Pediatr Dermatol 1996;13(6):477-82.

[24] Kavanagh GM, Colaco CB, Kennedy CT. Juvenile dermatomyositis associated with partial lipoatrophy. J Am Acad Dermatol 1993;28(2 Pt 2):348-51.

[25] Mukamel M, Horev G, Mimouni M. New insight into calcinosis of juvenile dermatomyositis: a study of composition and treatment. J Pediatr 2001;138(5):763-6. 
[26] Bingham A, Mamyrova G, Rother KI, Oral E, Cochran E, Premkumar A, et al. Predictors of acquired lipodystrophy in juvenile-onset dermatomyositis and a gradient of severity. Medicine 2008;87(2):70-86.

[27] Sanner H, Sjaastad I, Flatø B. Disease activity and prognostic factors in juvenile dermatomyositis: a long-term follow-up study applying the Paediatric Rheumatology International Trials Organization criteria for inactive disease and the myositis disease activity assessment tool. Rheumatology 2014;53(9):1578-85.

[28] Brown RJ, Chan JL, Jaffe ES, Cochran E, DePaoli AM, Gautier JF, et al. Lymphoma in acquired generalized lipodystrophy. Leuk Lymphoma 2016;57(1):45-50.
[29] Fredi M, Bartoli F, Cavazzana I, Ceribelli A, Carabellese N, Tincani A, et al. Calcinosis in poly-dermatomyositis: clinical and laboratory predictors and treatment options. Clin Exp Rheumatol 2017;35(2):303-8.

[30] Baechler EC, Bilgic H, Reed AM. Type I interferon pathway in adult and juvenile dermatomyositis. Arthritis Res Ther 2011;13(6):249.

[31] Brehm A, Liu Y, Sheikh A, Marrero B, Omoyinmi E, Zhou Q, et al. Additive loss-of-function proteasome subunit mutations in CANDLE/PRAAS patients promote type I IFN production. J Clin Invest 2015;125(11):4196-211. 